\title{
HOW TO TRANSLATE A VERBAL THEORY INTO A FORMAL MODEL
}

\author{
PAUL E. SMALDINO*
}

July 6,2020

\begin{abstract}
Turning verbal theories into formal models is an essential business of a mature science. Here I elaborate on taxonomies of models, provide ten lessons for translating a verbal theory into a formal model, and discuss the specific challenges involved in collaborations between modelers and non-modelers. It's a start.
\end{abstract}

6 Keywords: formal models; agent-based models; theory; SIR; flatten the curve

\section{INTRODUCTION}

Social, behavioral, and cognitive scientists study integrated systems of vast complexity. 9 The phenomena we're interested in involve time scales from evolutionary to historical, from developmental to synaptic; at spatial scales from the molecular to the planetary; and at levels of organization from the physiological to the cultural. Our charge is a 12 daunting one: to describe the behavior of a complex system in such a way that is meaningful for explanation and prediction.

The social, behavioral, and cognitive sciences have, historically, relied on the power 15 of the word. Words are powerful. Rich analogies can resonate in the minds of readers, appearing to illuminate the mysteries of nature. I'm talking about verbal theoriesdescriptive explanations of complex phenomena. Most theories are probably more work8 manlike than poetic, but they generally rely on a property of most languages, whereby phrases can carry several possible implicatures - consider, for example, that words like "perception," "category," "identity," "learning," and even "response" are sufficiently 21 ambiguous to allow for a multiplicity of interpretations. That is, language is inherently (and adaptively) vague and ambiguous (Grice, 1975; Eisenberg, 1984; Lee \& Pinker, 2010; Smaldino et al., 2018). This is ultimately a problem for scientists, because we 4 need to be exceptionally clear regarding what we are talking about in order to advance useful theories of the universe.

To solve this problem, we need formal models - mathematical or computational models 27 of complex processes. All mature sciences use formal models to develop, test, and extend theory. Models necessarily simplify and hence ignore many of the nuanced details of the real world, but this is a feature rather than a bug. Much has been written on the importance of formal models in the human sciences and related fields (Haldane, 1964;

* Department of Cognitive and Information Sciences, University of California, Merced

E-mail address: psmaldino@ucmerced.edu. 
Levins, 1966; Wimsatt, 1987; Varian, 1997; Bedau, 1999; Schank, 2001; Turchin, 2003; Bryson et al., 2007; Epstein, 2008; Weisberg, 2012; Nowak et al., 2013; Gunawardena, 3 2014; Servedio et al., 2014; Morecroft, 2015; Smaldino et al., 2015; Eberlen et al., 2017; Smaldino, 2017, 2019a; Page, 2018; Jolly \& Chang, 2019; Muthukrishna \& Henrich, 2019; Gervais, 2020; Guest \& Martin, 2020; Robinaugh et al., 2020; van Rooij \& Baggio, 2020), and I will not repeat those arguments here.

The question I tackle in this paper is: What comes next? You've come to appreciate the value of modeling, or you're at least model-curious. So how you do you turn your explains some phenomenon or pattern to a formal model that illustrates with precision how those mechanisms might work?

\section{Getting started}

This is an impossible question to adequately answer in a short paper like this one. Model development is very much an art form, and part of any art training is mastery of the fundamentals. Getting experience with reading, building, and analyzing many different models can provide us with a mental arsenal of parts and ideas that can be used and recombined for novel models. I don't know of any real shortcut there. I think haste makes for bad modeling. While I'm sure there are counterexamples of excellent models being made by dilettantes, I'm also sure that this is the exception rather than the rule. Becoming an expert modeler is like becoming an expert in anything: it takes time and dedication.

That said, I do think that there are some lessons I can distill from my own experience as a modeler to get you started. And that's the important thing: getting started. To some extent, it doesn't even matter what the model is at first (though choosing a model of something you care about will help you stay engaged). There's a lot to be said for simply playing around with model systems without any concrete goals. Grab some model code or jot down some relational equations and start messing around. Doing this is nontrivial, of course, bu luckily "grabbing some model code" is easier than ever. The modeling software NetLogo, for example, comes with an excellent library of model code, 0 and far more code can be found on repositories like GitHub and Modeling Commons in a variety of programming languages. Many published modeling papers also include links to source code.

An excellent way to get a feel for modeling is to begin with the code for a published model. It is ideal if you code this yourself (that is, replicating the model), but you can also use someone else's code as long as you take the time to understand how each line of 6 code contributes to the whole. Consider the model's explicit and implicit assumptions. If the model involves a population of individuals, is the population size fixed, or dynamic? Is the population structure well-mixed, or a network? How are initial traits distributed?

69 How do individuals make decisions? Think about alternative assumptions one could make, and implement some of them. Just getting a feel for how the dynamics work under varying assumptions is quite valuable; it is a skill that translates not only from one model to another, but also from models to real life. This sort of exercise will also 
help you get a sense of how to analyze models, by considering the questions you want to answer and the results you will accept as convincing. I would caution against diving immediately into trying to model your our own theories before you've cultivated this experience. There is, as in all things, a temptation to take shortcuts in one's analyses when they seem to support your pre-existing ideas. Probing someone else's work allows us to retain emotional distance and critical judgment. Try to retain your critical eye once you start building models of your own.

Let's talk about that: building a model for your own theory. I will tend to write as if you have a well developed verbal theory of something and you are interested in translating it into a formal model. In reality, the process of modeling can contribute directly to the process of theory development (Haldane, 1964; Wimsatt, 1987; Smaldino et al., 2015; Edmonds et al., 2019). What sort of model should we start with? What types of models are there, and how shall we classify and contrast types of models? A few words on this.

\section{TYPES OF MODELS}

A taxonomy is a means of dividing a class of objects into useful subclasses. As such, a taxonomy is itself a model for the relevant aspects of the superordinate class. There are probably many ways to create a taxonomy of models, but I'll focus on two distinctions I think are particularly useful.

3.1. Equation-based and computational models. One distinction people sometimes make is between equation-based and computational models (such as agent-based models) (e.g., Smith \& Conrey, 2007). Equation-based models involve writing down, well, equations that specify the key relationships between the parts of a system, such as the dynamics of how a population changes. In population models, classes of objects or individuals are treated as aggregates for the sake of tractability. Equation-based models can provide quite a bit of precision as well as a certain kind of mathematical elegance. Exploration of parameters is generally quite easy, since we can simply plug new numbers into the equations, and we can often derive the exact conditions under which particular outcomes will or will not occur. Even when precise, closed-form solutions are not possible, equation-based models can be explored through straightforward numerical simulation.

Equation-based models are limited primarily in their ability to deal with heterogeneity.

105 For example, we may want to explore the spatial or network structure of a population, or keep track of how individual differences in traits or behaviors are distributed; such things are challenging with equations only. In cases where additional complexity is desired and analytical tractability is not feasible (or is beyond the mathematical ability of the modeler), computational models can provide a useful alternative.

Agent-based models (ABMs) are a particular class of computational models in which individual agents (which are often meant to represent people, but agents can also represent anything from biological cells to economic firms to political municipalities) are simulated as explicit computational entities (Epstein, 1999; Bryson et al., 2007; Smaldino et al., 2015). In addition to allowing for greater heterogeneity, agent-based models have 
other advantages. One is that learning to code ABMs often represents a lower bar to entry than learning the requisite mathematics for analyzing equation-based models, especially for those with less formal mathematical training (not uncommon in the human sciences). A related advantage is that ABMs may provide the sort of intuitions for the behavior of complex systems that typically comes only from direct observation. For those without strong mathematical training, equations can be opaque or cryptic. Observing the behavior of agents in a visualized simulation can also help accomplish something that is often difficult to do with equations: confer understanding upon non-modelers.

In the interest of getting you more deeply into a modeling frame of mind, I am going to walk you through equation- and agent-based versions of a simple epidemiological model of disease transmission ${ }^{1}$. Readers more interested in general modeling advice may wish to skip to the end of this section. Consider the scenario where an infectious disease has broken out, so that individuals can be characterized as either susceptible to the disease $(\mathrm{S})$, infected (I), or recovered (R) and immune (or, alternatively, removed from the population for more depressing reasons). This is the well-known SIR model (Kermack \& McKendrick, 1927), the dynamics of which can be expressed as three coupled differential equations:

$$
\begin{aligned}
\frac{d S}{d t} & =-\beta S I \\
\frac{d I}{d t} & =\beta S I-\gamma I \\
\frac{d R}{d t} & =\gamma I
\end{aligned}
$$

These equations define the rates at which the relative numbers of susceptible, infected, and recovered individuals change over time, and represent two propositions about disease contagion. First, that susceptible individuals become infected via contact with infected individuals, at a rate that is proportional to the expected number of interactions between susceptible and infected individuals, tempered by the transmissibility of the infection, $\beta$. Second, that infected individuals recover at a constant rate, $\gamma$. An implicit assumption is that the rate of interactions between individuals in different states is exactly proportional to the frequencies of those states in the population - that is, that the population is "well-mixed." This model is simple, but powerful. It can be used to estimate the time course of an epidemic, the maximum number of infected individuals at a given time, and the number of individuals requiring immunity (such as by vaccination) needed to prevent an outbreak from becoming an epidemic, thereby providing "herd immunity." Variations on the model have considered a number of other factors, including non-contagious periods after exposure, age-structured populations, non-random assortment, and even simultaneous "behavioral contagions" that could alter transmission rates (Funk et al., 2010).

\footnotetext{
${ }^{1}$ The first draft of this paper was written under lockdown during the COVID-19 pandemic, so such models are very much on my mind.
} 
Beginning in the first months of the COVID-19 pandemic in 2020, authorities urged people to maintain physical distance from one another in order to "flatten the curve" of the epidemic. This meant that reducing physical contact would decrease the effective transmission rate of the disease and, critically, reduce the number of individuals infected at any given time. This is illustrated by numerically simulating the differential equations in the SIR model above for different values of $\beta$ (Figure 1). Although articles explaining this curve-flattening process proliferated, my personal experience was that many people did not find it intuitive that individual behaviors could translate to reduced transmissibility on a large scale.

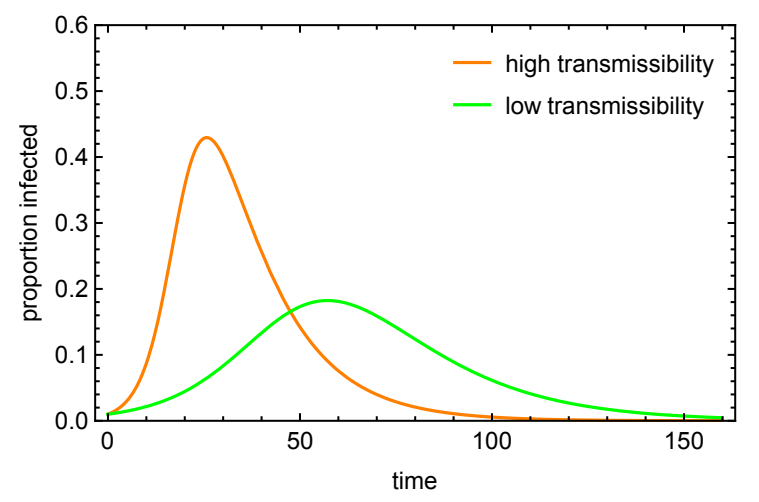

Figure 1. Temporal dynamics of infected individuals in the equationbased SIR model with a recovery rate of $\gamma=0.07$. This compares populations under either high transmissibility $(\beta=0.3)$ or low transmissibility $(\beta=0.15)$.

To provide an alternative framing, I built a simple agent-based model of SIR dynam$\mathrm{ics}^{2}$, in which agents are situated on a two-dimensional space and move around using a random walk. Anytime a susceptible individual is sufficiently close to an infected individual, they become infected with some probability (the transmission rate). An infected individual then recovers with a probability dictated by the disease's recovery rate (Figure 2a). Rather than modifying the disease transmission rate directly, as in the equation-based model, I modified the size of the step taken by agents during their random walks, so that they took either large steps (thereby rapidly traversing the space and interacting with many different individuals) or small steps (thereby staying close to where they started and interacting with a smaller number of distinct individuals; Figure $2 \mathrm{~b}$ ). Comparing the dynamics of the infected populations in these two movement conditions produces a plot that is comparable to that produced with the equation-based model $^{3}$, but illustrates more directly how a reduction in social contact flattens the curve

\footnotetext{
${ }^{2} \mathrm{~A}$ more detailed discussion of this model, as well as link to the NetLogo code, can be found at http://smaldino.com/wp/covid-19-modeling-the-flattening-of-the-curve/.

${ }^{3}$ I made no attempt to keep the transmission and recovery rates the same between the two models - my purpose here is to illustrate how both models can produce the same qualitative patterns.
} 
(Figure 3). It also reveals that "transmissibility" in the equation-based SIR model is an aggregate variable that incorporates properties of both the disease and its hosts.
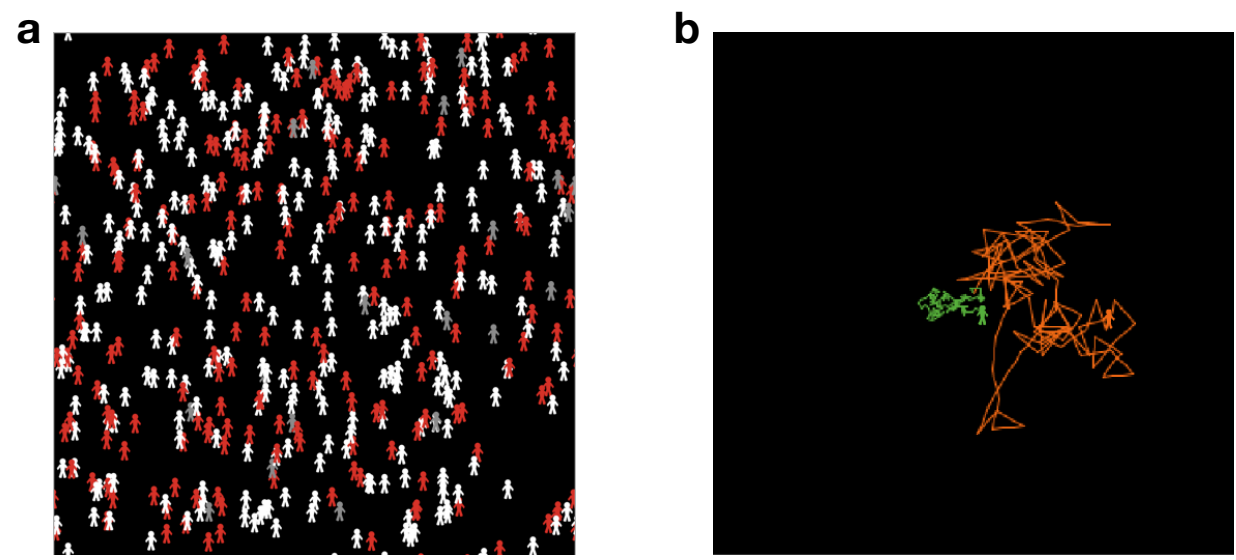

FiguRE 2. (a) Visualization of a spatial agent-based SIR model. There are 500 agents, which can be either susceptible (white), infected (red), or recovered (grey). (b) Example random walk trajectories over 100 steps for agents taking either large (orange) or small (green) steps.

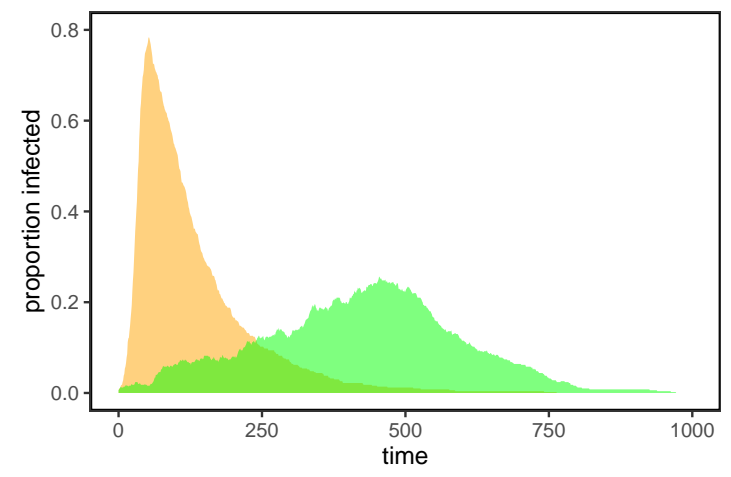

FiguRE 3. Temporal dynamics of infected individuals in the agent-based SIR model. Agents move using a random walk with either a large (orange) or small (green) step size.

I want to make it clear that both computational (agent-based) models and purely equation-based models are valuable, and attempts to paint them as competing techniques are misguided ${ }^{4}$. Both techniques are part of the modeler's toolkit, and one method is

\footnotetext{
${ }^{4}$ Indeed, the distinction is more heuristic than technical. Equation-based models can be explored computationally, and even complex agent-based models can, at least in theory, be reduced to a set of recursive mathematical functions (Epstein, 1999; North, 2014).
} 
not inherently superior to the other. In many cases, it can be valuable to combine both analytical equation-based models and agent-based simulations to provide richer coverage of the model system.

3.2. Fine-grained and coarse-grained models. Another taxonomy worth discussing is the distinction between what I will call fine-grained and coarse-grained models. Finegrained means that there are data in the world that can be used to precisely parameterize and test the models. Many models in physics are like this; the parameters of the models can be precisely measured quantities like mass, pressure, or voltage. In epidemiology, some agent-based models are calibrated using precise data on demographics, geography, schools, travel matrices, and so forth, with the goal of predicting the exact time course of an epidemic. In neuroscience, precise biophysical models might exactly predict the dynamics of action potentials or motor behaviors.

In the social and behavioral sciences, most models are more coarse-grained (the SIR models in the last section are examples of coarse-grained models). These models focus on broad, qualitative patterns in the data, not on reproducing exact measurements. There are at least three reasons for this. First, we may not need to be precise about the behavior of individuals if we can estimate the distributions of their behaviors at least 3 somewhat accurately. It is a long-standing principle of statistics that hard-to-predict things may become predictable when considered in aggregate, as long we know enough about how interactions are structured and how behaviors are distributed.

Second, measurement in the social and cognitive sciences is often very difficult. Processes related to cognition, behavior, and social organization involve interacting parts at many levels of organization and time scale. While the simpler sciences have focused their study on things that are readily measured like mass and motion, the social and cognitive sciences are concerned with emergent phenomena like emotions, perceptions, and norms. These concepts are formed in minds for their predictive power in lay thought and communication - and their utility in those domains is indisputable - but it is less obvious how they should be measured for scientific study. Even when a concept is precisely defined, measurement is often made difficult by constraints of time, resources, or ethics.

Third, complex systems are by their very nature difficult to model with great precision. The physicist-turned-social scientist Duncan Watts puts it this way:

Nobody really agrees on what makes a complex system "complex" but it's generally accepted that complexity arises out of many interdependent components interacting in nonlinear ways. The U.S. economy, for example, is the product of individual actions of millions of people, as well as hundreds of thousands of firms, thousands of government agencies, and countless other external and internal factors, ranging from the weather in Texas to interest rates in China. Modeling the trajectory of the economy is therefore not like modeling the trajectory of a rocket. In complex systems, tiny disturbances in one part of the system can get amplified to produce large effects somewhere else. ... When every tiny factor in a complex system can get potentially amplified in unpredictable ways, there is 
only so much that a model can predict. As a result, models of complex systems tend to be rather simple - not because simple models perform well, but because incremental improvements make little difference in the face of the massive errors that remain. (Watts, 2011, pp. 141-142)

Modeling complex systems is challenging, but challenging is not the same thing as futile. Far from it, in fact. As far as I'm concerned, the only alternative to using a formal model is to use a verbal model, or worse, an unspoken mental model. In those cases it is much more difficult to identify implicit assumptions or show how the explicit assumptions lead to particular consequences, and therefore much easier to enter into the territory of unscientific vagueness. Everyone is using some model; but it is hard to know how good that model is without writing it down. OK then. How do we write them down?

\section{TEN LESSONS For translating a VERbal theORY into A FORMAL MOdEL}

4.1. Develop relevant skills. Learning to model is like any other art form. There are myriad technical skills involved in doing it well. Not every practitioner is equally an expert in all techniques, of course, which is as it should be. We all work to our strengths. Moreover, certain techniques may be particularly useful for modeling certain processes. General strengths in mathematics, computer programming, and data visualization are always useful and worth cultivating. I appreciate that for many people, learning to code is not trivial. Unfortunately, there's just no getting around it. Today's scientists need at least some familiarity with coding. It doesn't much matter which programming language you use. I have personally published models written with Java, Python, NetLogo, and Mathematica, and I have colleagues who have published excellent modeling work using Javascript, R, C++, MATLAB, and Julia. All of these languages have their pros and cons, but each will get the job done, so I recommend using whichever is most convenient or appealing.

For those interested in modeling social processes, there are some technical domains that I have found particularly useful. These include game theory, evolutionary dynamics, dynamical systems, probability distributions, Bayes' theorem, network theory, connectionism, and information theory. In addition, there are some models that should probably be in every modeler's toolbox. Scott Page (2018) has recently provided an excellent primer on many of these models, and I refer you to his book. In general, one of the most important attributes you can possess is the confidence that you can learn new skills.

4.2. Be mindful of the literature. As with any scholarly endeavor, we want to make sure we are neither reinventing the wheel nor failing to give credit to those who blazed the trail ahead of us. For a modeling project, due diligence requires consideration of both the relevant modeling literature and the relevant empirical and theoretical literatures. The modeler is a scientist using a particular tool to ask scientific questions. Therefore, they need to engage with the same deep knowledge of their study system as would be expected of any other researcher working on that system using other means. 
This is also a call for a positive attitude toward interdisciplinarity. For example, let's say you are a social psychologist, and as such are interested in things like cooperation, coordination, signaling, social influence, and norms. There are well-developed modeling traditions related to these topics, often written by researchers and placed in journals that may be marked with different disciplinary labels, including biology, anthropology, sociology, economics, computer science, mathematics, or philosophy. These sciences are deeply interrelated. I once had a conversation with someone in a philosophy department during which we discussed their current modeling project. I mentioned what I considered relevant papers written by sociologists. He dismissed them, saying something like "I'm publishing in philosophy journals, and they're not going to care about these references." Please don't do this sort of thing. Your responsibility is, I hope, to produce and disseminate knowledge into the world rather to please the momentary gatekeepers of your subdiscipline.

I want to make it clear that my promotion of an interdisciplinary approach doesn't stem merely from some idealistic notion that interdisciplinarity is good, full stop. I think an interdisciplinary mindset yields tangible benefits by providing connections that facilitate better models of complex systems, and hence better theories about their workings. Here are just a few examples. Drawing on insights from the physics of magnetic spins, Hopfield (1982) built a model that showed how neural networks can recall stored memories from partial information. Drawing on insights from the study of epistatic gene networks, Lazer \& Friedman (2007) built a model that showed how social network structure influences the performance of teams working to solve collective problems. Drawing on insights from ecology and dynamical systems theory, Turchin $(2003,2016)$ built a model that showed how interconnections between the general population, elites, and government can contribute to cycles of political stability and instability. Drawing on insights from infectious disease modeling, several researchers have explored how innovations, products, and behaviors diffuse, showing that social transmission may work in similar - though not identical — ways as disease transmission (Bass, 1969; Centola, 2018). The interdisciplinary field of cultural evolution, perhaps the best candidate for a uni9 fying framework for understanding human behavior (Mesoudi, 2017; Muthukrishna \& Henrich, 2019), relies on models that draw from evolutionary ecology, social psychology, anthropology, and economics. In sum, other disciplines have developed useful techniques. 2 Don't ignore them. Insights can come from anywhere.

While we should acknowledge and learn from what has come before, we should also avoid being a slave to the past. The way we model a system constrains the questions 5 that we can ask about that system (Smaldino, 2019b). Therefore, just because a system tends to be modeled in a particular way doesn't mean that other decompositions aren't valuable.

4.3. Decide on the parts of the system. For over 25 years, John Miller and Scott Page have run a summer workshop at the Santa Fe Institute on computational modeling for graduate students in the social sciences. An exercise they usually give at the start

291 of the workshop is a modeling task that is deliberately fuzzy on the details. When I attended their workshop in 2008, the task was as follows: 
People enter and leave an elevator as it travels up and down. Model, using whatever techniques you wish, the above scenario. Explicitly state your model and key assumptions. Summarize key results. Suggest some potentially interesting future directions and questions for the model. Suggest some standard social science scenarios that could be usefully modeled using such a process.

We split into small groups of two or three, and spent a day working on the project. What emerged was fascinating to me. My group had focused on the decisions made by individuals needing to move between floors as to whether to take the elevator or the stairs. The calculus was based on the distance required to travel, the time of day, and the number of people currently in the elevator ${ }^{5}$. It was a zoomed-out view where the relevant parts were the location and destination of the individual decision maker and the location and fullness of the elevator. Other groups focused on entirely different aspects of the problem. One that sticks out in my memory was a group that modeled optimal ways for agents to arrange themselves within an elevator so that they avoided crowding, while also minimizing the likelihood of being blocked in when the elevator reached their floor. Two completely different approaches to the same modeling prompt.

The reason such vastly different model designs could emerge from the same prompt is that although the elevator scenario is a reasonably well-defined system, the prompt provides no specific questions to be addressed. It is the question that determines the relevant parts of the system. To formulate a scientific theory or hypothesis about some system, it is necessary to decompose that system into relevant parts, their properties, and the relationships between them (Kauffman, 1971). There is no one right way to do this for a given system; rather, the value of a particular decomposition derives from its ability to answer meaningful questions in useful ways. A model is an instantiation of these parts and relationships. Because there are lots of ways to represent any particular system, you need to think carefully about the parts you are going to focus on, and the parts you are ignoring. What questions does your verbal theory address? What parts do you need to include to answer those questions? Is your representation a satisfying analogue to your verbal theory? If not, why not?

Such a consideration may also highlight limitations to your verbal theory. I was once involved in a project where the task was to produce a model of a psychologist's theory of emotions. I pored over their papers to understand how the theory worked, with an aim to see how it might be instantiated in a formal model. I realized, however, that the theory was based entirely on explaining the internal phenomenology of emotional experience, and had not specified how emotional responses should influence behavior. This created a significant challenge for the construction of a model, because it was never clear what sort of behavior on the part of the model would be consistent or inconsistent

\footnotetext{
${ }^{5}$ Our solution is, miraculously, still available online at http://jhmsfi.com/econ/homework08/2/ index.html.
} 
with the theory. Modeling can help us not only test but develop our theories by forcing us to consider each assumption - and each consequence - explicitly ${ }^{6}$.

4.4. Separate design from construction. In his book, Gödel, Escher, Bach: An eternal golden braid, the cognitive scientist Douglas Hofstadter (1979) introduced Hofstadter's Law: It always takes longer than you expect, even when you take into account Hofstadter's Law. This is a good lesson for almost any complex project, and certainly one for modeling projects. The truth is that once you learn some math and pick up some coding chops, it often doesn't take very long to program a model. This can lead to the mistaken impression that modeling doesn't take very long. The error is to conflate the programming with the modeling. The hardest part of modeling is almost always designing the model, figuring out how the whole thing works. This process can take ages, with lots of false starts and returns to the drawing board.

This is why I usually encourage modelers to write out their entire model before a single line of code is written. That is, you should separate the process of model design, in which you figure out all of your assumptions and how they will be implemented, from the process of model construction, in which you actually code the thing so it works. If you are building a house (or a boat, or a cabinet, or a guitar, or whatever DIY project resonates with you), you know you shouldn't start hammering before making sure you've got all your parts, all your tools, and have laid out your plans. If you do start before these things are done, you know you are more likely to make wrong turns, paint yourself into corners, and find yourself committed to unfortunate choices. In practice, I find that the design stage often works best as an iterated process with multiple steps: going from verbal theory to a set of parts and relationships, to a set of parameters, to a set of analyses, to an algorithmic design in pseudocode, and then finally to a coded model. All the while, be willing to reconsider the choices you've made in each step.

4.5. Be as simple as you can be, as complicated as you need to be. A model gets its power from its simplicity, from its ability to remove complexity from the world, which allows us to focus only on those aspects we think matter (Smaldino, 2017). Many of the most powerful models in the cognitive, behavioral, and social sciences are simple models that reveal powerful and counterintuitive dynamics. I have seen extremely complicated models that merely reproduced the insights of simpler models with less transparency and generalizability. An important but overlooked fact is that simple models are not 3 necessarily easier to produce! It's often much easier to produce a complicated model ${ }^{7}$. I have certainly had the experience of starting a modeling project with a very complicated design. As I developed a deeper understanding of theory I was trying to model, I was 6 able to see more clearly the parts of the system that were critical to that theory, as well as the parts that I could, at least for the moment, leave out (Smaldino et al., 2019). It is a common experience among modelers to have non-modelers ask for more realism in 69 the model design, but giving in to these requests is often a mistake. As the sociologist

\footnotetext{
${ }^{6}$ This should also serve to remind us cognitive processes are selected for (by learning, biology, or cultural evolution) by virtue of their influence on behavior.

${ }^{7}$ Recall Blaise Pascal's famous quip: "I have made this letter longer than usual because I have not had time to make it shorter."
} 
Kieran Healy (2017) points out, it is actually by establishing limits that models allow for the creative development of new ideas.

The economist Hal Varian has described this phenomenon quite well:

Several years ago I gave a seminar about some of my research. I started out with a very simple example. One of the faculty in the audience interrupted me to say that he had worked on something like this several years ago, but his model was "much more complex." I replied "My model was complex when I started, too, but I just kept working on it till it got simple!" And that's what you should do: keep at it till it gets simple. The whole point of a model is to give a simplified representation of reality. (Varian, 1997, p. 4)

This is not to say that simple models are always best. While you should strive to keep your model simple, don't be afraid to get complicated when the situation calls for it. Added complexity creates new affordances for the your model's behavior. Heterogeneity and structure create new opportunities for feedback. Once you understand the simple cases, adding complexity to a model can yield important insights that would otherwise be missed. Examples can be found in the fields of artificial life (Bedau, 2003) and systems science (Meadows, 2008; Morecroft, 2015), which are known for embracing complicated computational models to explore how the myriad components of a complex system interact to produce emergent outcomes.

4.6. Attack your design. Once you've designed your model and articulated the relevant parts and relationships, attack it! Seek out its weaknesses and push them until the whole thing falls apart. You need to know its limitations. A model can be viewed as a metaphor for your study system, and as the biologist Richard Lewontin was fond of saying, "the price of metaphor is eternal vigilance" (Lewontin, 1981).

It's also worth considering the modeling framework you've employed. If you are working with a mathematical model, are there frustrating simplifications for which an agent-based or other computational approach would shed light? If you are using a computational model, are there findings that could be expressed more simply and cleanly, perhaps even conclusively proved, using a mathematical model? Mathematical and computational approaches are often complementary, and can be usefully employed to explore variations on the same model.

4.7. Plan your analyses. A critical consideration in designing a model is what the outcome measures will be. This forces us to ask again: what are we trying to show? What are the questions we want answered about our system? These can then be reframed as: How do you structure your model so that it answers these questions? Are you interested in the state of the model at equilibrium? The frequency of a trait or the solution to a problem? Are you more interested in dynamics, the rise and fall of some variable? Will you need to consider the variation among the agents in your model, or the variation of outcomes between simulation runs?

The challenge of coming up with good questions should not be underestimated, and these considerations should be prominent in your mind. As noted, I find it extremely 
valuable to write out a formal, detailed description of the model before any analyses or simulations are performed. That way, I make sure that I (as well as any collaborators) am extremely clear on how the model works. The description also provides a record of what you were intending to do, which is important as model designs can otherwise change as decisions are made on the fly while coding. A critical component is a detailed 47 description of outcome measures, particularly if you are working with a computational model, for which the possible analyses are more numerous. What are the parameters and initialization conditions you will vary? If your model is very complicated, how will you 420 deal with the issue of many interacting variables? The more complicated your model, the larger the space of possible parameter combinations. You will need to think carefully about how you will cover this space, given that once your model has grown past a few parameters, a full sweep of all permutations becomes logistically tricky.

4.8. Rethink statistics. Suppose you are working with a simulation model, and you have some nice results from your many simulations. How shall you describe the ways in which your outcome measures interact with your model parameters? If you have been trained in the traditions of most of the behavioral and social sciences, you will likely be tempted to use inferential statistics such as regression ${ }^{8}$. Although there are exceptions, this is almost never the right decision (White et al., 2014; Smaldino, 2017).

Why? Surely you want to know if the effects you are seeing are valid? Of course. But consider that calculating inferential statistics involves constructing a model of your datagenerating process, usually with strong simplifying assumptions about the distributions of parameter values. You don't need to do this, because you already have a better model of your data-generating process: it's your actual model. There's no reason to model your model with a shittier model.

This is true even if your model is extremely complicated, so that understanding the mechanisms underlying its behavior is difficult. Rather than estimating the likely distributions of data produced by the model by appealing to general statistical properties of large data sets, you can simply run enough simulations to obtain arbitrarily precise estimates. I have heard some modelers complain about this suggestion, because running many simulations can take a long time, particularly if one does not have access to a computing cluster. I sympathize. However, we are doing science here, and our job is to be get as close as possible to the truth ${ }^{9}$. Think about your colleagues who spend months or years doing fieldwork or collecting longitudinal data. Surely in the interest of precision you can wait a few extra days while your simulations run.

4.9. Get your story straight. Think hard about what you are trying to accomplish with your model. What are you trying to show? A model is built around an often-implicit story. Once there was an infection that arose in some people, and spread from person to person. Or, once there were two groups of people who lived in a city, and each moved when there weren't enough people from their own group living nearby (Schelling, 1971).

\footnotetext{
${ }^{8}$ The anthropologist Bret Beheim once said to me, "All statistics are descriptive statistics," which is a fair point.

${ }^{9}$ This a placeholder for your preferred caveat about the nature of truth and its relationship with the human mind.
} 
Or, once there was a land in which there were two norms of behavior, and people faced the challenge of communicating their norm to potential relationship partners (McElreath 3 et al., 2003). Getting your story straight is extremely important, because it will help you figure out what aspects of reality you are putting into your model, and therefore what aspects you can safely leave out. Understanding your story also becomes useful when 6 you write up your model for dissemination. Describing the story of the model is a huge help in interpreting the mathematical or computational details of the model, because the story provides a map that shows what each component is intended to represent.

4.10. Be open. Is this not the era of open science? Then let us be open. A model's value comes from its power to show how assumptions lead to consequences. If those assumptions are not transparent, then you have failed to truly offer a formal model. Instead, you have merely added alchemy to your verbal theory. Your model should be described clearly. I hold to the standard that a competent modeler who is otherwise unfamiliar with your project should be able to read your paper (or its appendix, or wherever you have placed the full model description) and, without looking at your source code, be able to produce their own working version of the model. I sometimes have the impression that vague model descriptions come from modelers who are unsure of the wisdom of their choices.

Writing up a clear description of a complicated model is a skill that requires practice to hone. There are many good suggestions in the ODD protocol, widely used in ecology, for describing agent-based models (Grimm et al., 2010, 2020). The protocol suggests a three-stage strategy of model description: the Overview (the "story" of the model), the Design (the computations involved in the model's mechanics), and the Details (all the algorithmic details, sometimes relegated to an appendix). Even if I am unsure that a generic protocol that is appropriate for describing any model design is possible, there are nevertheless many good and widely applicable ideas in the ODD framework.

While you should be clear in your description, you should also share your code. The code should be well commented, and readily available on a stable repository ${ }^{10}$. There are at least two reasons to do so. First, even the best writers sometimes unintentionally 40 inject ambiguity, and this can be cleared up by referencing the source code. Second, sharing code provides a service to those who wish to build on your work. Some people are reluctant to simply give away their hard-earned source code. Personally, I have never 3 seen anyone's career hurt by their sharing information. Your influence can only increase when others can readily build on your work.

\section{LESSONS FOR MODELING COLLABORATIONS}

I believe every researcher in the social, behavioral, and cognitive sciences should develop the expertise to engage with and learn from the modeling literature. Models are an essential part of science, and this is true even if you never build a model yourself. Because of this, there are many opportunities for fruitful collaborations between modelers and empirical researchers. Such collaborations are also fraught with peril, because the

\footnotetext{
${ }^{10}$ Please don't succumb to the dodgy practice of saying that the code is "available upon request." The success rate of such requests is notoriously low.
} 
languages of the different research traditions can lead to misunderstanding and resentment. This can be avoided, I think, by considering carefully what each type of expert brings to the project.

For modelers, the first step is to take the project seriously. The empirical researchers have real skills and real knowledge. If people have known about a phenomenon for more than a few years, there is a good chance that there is a deep literature on the subject and that many of the key problems have been identified or even solved. The modeler may think of him- or herself as an explorer of some uncharted land, mapping previously hidden territories with new tools. But there are natives in these lands, who have used their own tools to become intimate with its complex topography. These are the disciplinary experts, and only a fool would avoid the opportunity to learn from them (Miller, 1995). They can be vital to the project, shaping the model's assumptions and parameters. Your task is to ensure they have to opportunity to contribute the full value of their expertise, and this is done by making sure that they understand the modeling process, and especially how the model works, at a mechanistic level.

Speaking of expertise, if you are producing a model of some phenomenon, you must become an expert in that phenomenon. It is not acceptable to say "well, I'm not really a biologist" or "I'm not really a social scientist" or whatever, just because your training or appointment is in another discipline. If you are working on a model of biological processes, you are a biologist. If you are working on a model of social processes, you are a social scientist. You should therefore be judged as a member of those groups. Act accordingly.

For non-modelers, the first step is to take the project seriously. The modelers have real skills and real knowledge. They are not merely technicians, but scientists and designers. This is why simply hiring a programmer to develop your model is usually a mistake, except in situations where you are extremely clear as to how the model will be constructed and analyzed. The modeler must understand the science driving the research questions. Your task is ensure that this is the case. But here's the thing: you cannot do this if you do not understand how the model works. Every equation. Every algorithm. Every assumption so that the real-world system is decomposed into model parts that are meaningful, with properties and relationships that represent a reasonable instantiation of your theory. The biologist Jon Wilkins has made this point quite forcefully. The following quotation concerns collaborations between mathematicians and biologists, but is applicable to any collaboration between modelers and empirical researchers:

If you're [the biologist], you have to sit down with your mathematician, and you have to walk through every single equation. You have to press them on what it means, and you have to follow the thread of what it implies. If you're the mathematician, you have to sit down with your biologist and say, "If we assume A, B, and C, then mathematically that implies X, Y, and Z." You have to understand where, in the biology, A, $\mathrm{B}$, and $\mathrm{C}$ come from, and you have to work together to discover whether or not X, Y, and Z make any sense. Basically, each of you has to develop some fluency in the other's language, at least within the narrow domain 
covered by the collaboration. If you're not willing to put in this level of work, then yes, you should probably consider a different career. (Wilkins, 2013)

\section{Conclusion}

Once we get past the question of whether we should employ models in our science, the next question is how to do it. No single paper can answer that question in full, and probably no single book can either. Like many things, the best way to get started is just to start somewhere, and realize that you are going to make a lot of mistakes along the way. As you forge on, try to make the best models you can, but also remember not to let the perfect be the enemy of the good. Models are analogies, and all analogies are imperfect. They apply to only some aspects of a system and not to others. Understanding a complex system often requires a family of models that cover different aspects of the system. Don't overly concern yourself with producing the model of a phenomenon. Make a model. If you are diligent, the model's failures to match reality can tell you as much about your system as the model's successes (Wimsatt, 1987). Chances are, if you learned something from building and analyzing your model, others may benefit from hearing about it. Get crackin'.

\section{ACKNOWLEDGMENTS}

I thank Matt Zefferman, Liz Hobson, Jeremy Brooks, Tim Waring, Bret Beheim, Riccardo Fusaroli, Michael Muthukrishna, Igor Nikolic, Ted Pavlic, and Peter Turchin for comments on earlier drafts. I thank Hans IJzerman and Kai Epstude for inviting me to write a paper on modeling. I am indebted Jeremy Brooks for asking me whether there were any good resources on translating a vague verbal model into a more precise formal model, thereby providing me with a clear goal for the paper. Any failure to achieve that goal is entirely my fault.

\section{REFERENCES}

Bass, F. M. (1969). A new product growth for model consumer durables. Management Science, 15(5), 215-227.

Bedau, M. A. (1999). Can unrealistic computer models illuminate theoretical biology. In Proceedings of the 1999 genetic and evolutionary computation conference workshop program, (pp. 20-23). Citeseer.

Bedau, M. A. (2003). Artificial life: Organization, adaptation and complexity from the bottom up. Trends in Cognitive Sciences, 7(11), 505-512.

Bryson, J. J., Ando, Y., \& Lehmann, H. (2007). Agent-based modelling as scientific method: a case study analysing primate social behaviour. Philosophical Transactions of the Royal Society B: Biological Sciences, 362(1485), 1685-1699.

Centola, D. (2018). How behavior spreads: The science of complex contagions. Princeton University Press. 
Eberlen, J., Scholz, G., \& Gagliolo, M. (2017). Simulate this! An introduction to agentbased models and their power to improve your research practice. International Review of Social Psychology, 30(1), 149-160.

Edmonds, B., Le Page, C., Bithell, M., Chattoe-Brown, E., Grimm, V., Meyer, R., Montañola-Sales, C., Ormerod, P., Root, H., \& Squazzoni, F. (2019). Different modelling purposes. Journal of Artificial Societies and Social Simulation, 22(3), 6.

Eisenberg, E. M. (1984). Ambiguity as strategy in organizational communication. Communication Monographs, 51(3), 227-242.

Epstein, J. M. (1999). Agent-based computational models and generative social science. Complexity, 4(5), 41-60.

Epstein, J. M. (2008). Why model? Journal of Artificial Societies and Social Simulation, $11(4), 12$.

Funk, S., Salathé, M., \& Jansen, V. A. (2010). Modelling the influence of human behaviour on the spread of infectious diseases: a review. Journal of the Royal Society Interface, 7(50), 1247-1256.

Gervais, W. M. (2020). Practical methodological reform needs good theory. https: //psyarxiv.com/jcs6e.

Grice, H. P. (1975). Logic and conversation. In P. Cole \& J. L. Morgan (Eds.), Syntax and semantics Vol. 3: Speech acts (pp. 41-58). Academic Press.

Grimm, V., Berger, U., DeAngelis, D. L., Polhill, J. G., Giske, J., \& Railsback, S. F. (2010). The ODD protocol: a review and first update. Ecological Modelling, 221(23), $2760-2768$.

Grimm, V., Railsback, S. F., Vincenot, C. E., Berger, U., Gallagher, C., DeAngelis, D. L., Edmonds, B., Ge, J., Giske, J., Groeneveld, J., et al. (2020). The ODD protocol for describing agent-based and other simulation models: A second update to improve clarity, replication, and structural realism. Journal of Artificial Societies and Social Simulation, 23(2).

Guest, O. \& Martin, A. E. (2020). How computational modeling can force theory building in psychological science. https://psyarxiv.com/rybh9/.

Gunawardena, J. (2014). Models in biology: 'accurate descriptions of our pathetic thinking'. BMC biology, 12(1), 29.

Haldane, J. B. S. (1964). A defense of beanbag genetics. Perspectives in Biology and Medicine, 7(3), 343-360.

Healy, K. (2017). Fuck nuance. Sociological Theory, 35(2), 118-127.

Hofstadter, D. R. (1979). Gödel, Escher, Bach: An eternal golden braid. Basic Books.

Hopfield, J. J. (1982). Neural networks and physical systems with emergent collective computational abilities. Proceedings of the National Academy of Sciences, 79 (8), 25542558.

Jolly, E. \& Chang, L. J. (2019). The Flatland fallacy: Moving beyond low-dimensional thinking. Topics in Cognitive Science, 11(2), 433-454.

612 Kauffman, S. A. (1971). Articulation of parts explanation in biology and the rational search for them. In R. C. Buck \& R. S. Cohen (Eds.), PSA 1970 (pp. 257-272). Philosophy of Science Association. 
Kermack, W. O. \& McKendrick, A. G. (1927). A contribution to the mathematical theory of epidemics. Proceedings of the Royal Society of London A, 115(772), 700721.

Lazer, D. \& Friedman, A. (2007). The network structure of exploration and exploitation. Administrative Science Quarterly, 52(4), 667-694.

Lee, J. J. \& Pinker, S. (2010). Rationales for indirect speech: the theory of the strategic speaker. Psychological Review, 117(3), 785.

Levins, R. (1966). The strategy of model building in population biology. American Scientist, $54(4), 421-431$.

Lewontin, R. C. (1981). On constraints and adaptation. Behavioral and Brain Sciences, $4(2), 244-245$.

McElreath, R., Boyd, R., \& Richerson, P. (2003). Shared norms and the evolution of ethnic markers. Current Anthropology, 44 (1), 122-130.

Meadows, D. H. (2008). Thinking in systems: A primer. Chelsea Green Publishing.

Mesoudi, A. (2017). Pursuing Darwin?s curious parallel: Prospects for a science of cultural evolution. Proceedings of the National Academy of Sciences, 114(30), 78537860 .

Miller, G. F. (1995). Artificial life as theoretical biology: How to do real science with computer simulation. https://www.primalpoly.com/s/ 1995-real-science-simulation.pdf.

Morecroft, J. D. (2015). Strategic modelling and business dynamics: A feedback systems approach. John Wiley \& Sons.

Muthukrishna, M. \& Henrich, J. (2019). A problem in theory. Nature Human Behaviour, 3(3), 221-229.

North, M. J. (2014). A theoretical formalism for analyzing agent-based models. Complex Adaptive Systems Modeling, 2(1), 3.

Nowak, A., Rychwalska, A., \& Borkowski, W. (2013). Why simulate? to develop a mental model. Journal of Artificial Societies and Social Simulation, 16(3), 12.

Page, S. E. (2018). The model thinker: What you need to know to make data work for you. Hachette.

Robinaugh, D., Haslbeck, J., Ryan, O., Fried, E. I., \& Waldorp, L. (2020). Invisible hands and fine calipers: A call to use formal theory as a toolkit for theory construction. https://psyarxiv. com/ugz7y/.

Schank, J. C. (2001). Beyond reductionism: Refocusing on the individual with individual-based modeling. Complexity, 6(3), 33-40.

Schelling, T. C. (1971). Dynamic models of segregation. Journal of Mathematical Sociology, 1(2), 143-186.

Servedio, M. R., Brandvain, Y., Dhole, S., Fitzpatrick, C. L., Goldberg, E. E., Stern, C. A., Van Cleve, J., \& Yeh, D. J. (2014). Not just a theory?the utility of mathematical models in evolutionary biology. PLoS Biology, 12(12).

Smaldino, P. (2019a). Better methods can't make up for mediocre theory. Nature, $575(7781), 9$.

Smaldino, P. (2019b). Five models of science, illustrating how selection shapes methods. https://doi.org/10.31235/osf .io/ghb4p. 
Smaldino, P. E. (2017). Models are stupid, and we need more of them. In R. R. Vallacher, S. J. Read, \& A. Nowak (Eds.), Computational social psychology (pp. 311331). Routledge.

Smaldino, P. E., Calanchini, J., \& Pickett, C. L. (2015). Theory development with agent-based models. Organizational Psychology Review, 5(4), 300-317.

Smaldino, P. E., Flamson, T. J., \& McElreath, R. (2018). The evolution of covert signaling. Scientific Reports, 8(1), 1-10.

Smaldino, P. E., Lukaszewski, A., von Rueden, C., \& Gurven, M. (2019). Niche diversity can explain cross-cultural differences in personality structure. Nature Human Behaviour, 3(12), 1276-1283.

Smith, E. R. \& Conrey, F. R. (2007). Agent-based modeling: A new approach for theory building in social psychology. Personality and Social Psychology Review, 11(1), 87104.

Turchin, P. (2003). Historical dynamics: Why states rise and fall. Princeton University Press.

Turchin, P. (2016). Ages of discord: A structural-demographic analysis of American history. Beresta Books.

van Rooij, I. \& Baggio, G. (2020). Theory before the test: How to build highverisimilitude explanatory theories in psychological science. https://psyarxiv.com/ $7 \mathrm{qbpr} /$.

Varian, H. R. (1997). How to build an economic model in your spare time. The American Economist, 41(2), 3-10.

681 Watts, D. J. (2011). Everything is obvious:* Once you know the answer. Crown Business.

Weisberg, M. (2012). Simulation and similarity: Using models to understand the world. Oxford University Press.

684 White, J. W., Rassweiler, A., Samhouri, J. F., Stier, A. C., \& White, C. (2014). Ecologists should not use statistical significance tests to interpret simulation model results. Oikos, 123(4), 385-388.

Wilkins, J. F. (2013). EO Wilson is wrong again-not about math, but about collaboration. http://jonfwilkins.com/2013/04/ e-o-wilson-is-wrong-again-not-about-math-but-about-collaboration/.

Wimsatt, W. C. (1987). False models as means to truer theories. In M. H. Nitecki \& A. Hoffman (Eds.), Neutral models in biology (pp. 23-55). Oxford University Press. 